\title{
Characterization of IL-17AA and IL-17FF in rheumatoid arthritis and multiple sclerosis
}

\begin{abstract}
Aim: IL-17 is thought to play a prominent role in immune disorders. Sensitive and specific IL-17AA and IL-17FF assays were developed and used to determine levels in serum and cerebrospinal fluid (CSF) from patients with rheumatoid arthritis and relapsing remitting multiple sclerosis (RRMS). Results: Qualified assays detected IL-17AA and IL-17FF in healthy and disease samples. Serum IL-17AA was significantly higher in rheumatoid arthritis and RRMS as compared with normal healthy subjects. IL-17AA was also elevated in RRMS CSF as compared with normal healthy subjects; although correlation was observed between serum levels of the two isoforms, no correlation was detected between serum and CSF levels. Conclusion: Reliable determination of IL-17 isoforms in the systemic and CNS compartments sheds light on the involvement of IL-17AA and IL-17FF in autoimmunity.
\end{abstract}

First draft submitted: 26 July 2016; Accepted for publication: 23 August 2016;

Published online: 13 September 2016

Keywords: CSF •IL-17A •IL-17F • multiple sclerosis • rheumatoid arthritis • serum

IL-17 is a proinflammatory cytokine that plays a key role in host defense against extracellular bacterial and fungal infections [1]. IL-17 recruits monocytes and neutrophils to sites of inflammation and is thought to be the central mediator of cellular immunity, regulating critical proinflammatory cytokines [2].

IL-17A and IL-17F are two of the six known members (A-F) of the IL-17 cytokine family with $50 \%$ sequence homology. Of the six, IL-17A and IL-17F are produced by Th17 $\mathrm{T}$ cells and other immune cells and are the most studied IL-17 isoforms [3]. Interestingly, although these dimers share similar receptors, evidence suggests that because IL-17A and IL-17F differentially regulate secretion of chemokines specifically in the presence of other proinflammatory cytokines, such as TNF- $\alpha$ and IL- $1 \beta$, they may not impact the inflammatory process equally [4]. IL-17 isoforms are secreted as $35-\mathrm{kDa}$ active homodimers (AA and FF), or as active heterodimers (AF) [5]. IL-17AA, IL-17FF and IL-17AF bind the same receptor complex comprising IL-17RA and IL-17RC subunits. IL-17AF heterodimers were reported to elicit a milder proinflammatory response as compared with the IL-17 homodimers [6].

There are three major subsets of IL-17-producing cells; Th17, ROR $\gamma \mathrm{t}^{+}$innate lymphoid cells (IL-17+ ILC3) and $\gamma \delta$ T cells [7]. In addition, innate immune cells, such as macrophages, are also reported to express IL-17AA and IL-17FF. There is evidence that regulation of secretion of IL-17AA and IL-17FF may depend on the microenvironment and may be distinct in different IL-17-producing cells [8].

The pathogenic properties of Th17 cells and their involvement in autoimmunity were recently reported [9], and increased production of IL-17 has been associated with several autoimmune disorders including psoriasis, rheumatoid arthritis (RA) and multiple sclerosis (MS) [10]. In RA, IL-17 contributes to cartilage destruction by stimulating
Cheryl Schofield', Saloumeh K Fischer', Michael J Townsend', Sofia Mosesova ${ }^{\ddagger 1}$, Kun Peng 1 , Alvernia Francesca Setiadi', An Song1 \& Amos Baruch ${ }^{* 1}$ ${ }^{1}$ Genentech, Inc., 1 DNA Way, South San Francisco, CA 94080, USA

*Author for correspondence:

Tel.: +1 6502554650

Fax: +1 6507425132

baruch.amos@gene.com ${ }^{\ddagger}$ Current address: Denali Therapeutics, 151 Oyster Point Blvd, South San Francisco, CA 94080, USA 
the expression of cartilage-degrading enzymes and to bone destruction by enhancing the expression of RANKL on osteoblasts that activate RANK-positive osteoclasts [11].

There is a substantial body of literature suggesting a role of IL-17 in MS. IL-17 is produced by infiltrating immune cells and can act on astrocytes and microglial cells to stimulate production of proinflammatory cytokines and chemokines. Previously, mRNA expression of IL-17 was detected in perivascular lymphocytes, as well as in astrocytes and oligodendrocytes located in the areas of active MS lesions. Furthermore, this was accompanied by a significant increase in the number of IL-1 $7^{+} \mathrm{T}$ cells in active lesions and not in MS lesions thought to be inactive [12].

Recently, IL-17A has generated significant interest as a therapeutic target for synovial inflammation and joint destruction in RA and as a potential therapy for MS. Several antibody therapeutics that inhibit IL-17A or its respective receptor have been evaluated in clinical trials, including secukinumab (Cosentyx $\left.{ }^{\circledR}\right)$, ixekizumab and brodalumab, which target IL-17 for RA and psoriasis [13-15]. Secukinumab is the first-in-class anti-IL-17 agent, initially approved for the treatment of plaque psoriasis, and more recently for the treatment of ankylosing spondylitis and psoriatic arthritis. Secukinumab has also shown promise as a therapy for MS supporting an active role for Th17 cells in MS [16]. Although there is mounting evidence that both circulating and central Th17 cells contribute to MS pathology, the relative contribution of different Th17 populations and the derived IL-17AA and IL-17FF isoforms is not fully understood.

As the interest in the biology of IL-17 continues to grow, accurately measuring the level of the circulating cytokine in clinical samples from patients with various autoimmune pathologies has become critical. Immunoassays used for the quantification of circulating IL-17 are still evolving, and a wide range of IL-17 levels have been reported in the literature using a number of assay platforms and reagents [17-25].

However, many of the assays used have limitations due to several factors: no evaluation of assay specificity was included; assay's sensitivity is often insufficient to accurately determine IL-17 in body fluids; careful evaluation of background signal was not performed and there is no inclusion of heterophilic antibody-blocking agents to reduce false-positive interference; and basic information on assay performance and characterization is lacking. In addition, it is often hard to determine which IL-17 isoform is being measured since this information is often lacking in published reports. As a result, reported serum values from normal healthy subjects (NHS) are highly variable and range from undetectable to $>100 \mathrm{pg} / \mathrm{ml}$. Only a few studies have appropriately characterized their assay, and their results suggest that circulating IL-17 levels are present at single-digit $\mathrm{pg} / \mathrm{ml}$ to sub-pg/ml levels in samples from normal subjects or in patients with chronic inflammatory conditions. For example, a well-qualified IL-17AA and IL-17FF ELISA method with an LLOQ of 1.6 and $12.3 \mathrm{pg} / \mathrm{ml}$ for IL-17AA and IL-17FF, respectively, failed to detect serum IL-17 from RA patients or NHS in any of the samples tested [20]. Consistently, reference values for serum IL-17AA in healthy subjects were recently published, using the Singulex highsensitivity platform, reporting upper 95 th percentile limit of $1.93 \mathrm{pg} / \mathrm{ml}$ with the majority of values registered at the sub-pg/ml level [24]. Less information is available on assays that can provide a reliable quantitation of IL-17FF in health and disease. For example, median serum levels of $20 \mathrm{pg} / \mathrm{ml}$ were previously reported for IL-17FF [17]. This is, however, in contrast to the report by DeForge et al. that could not quantify serum IL-17FF in any of the samples tested using a well-qualified assay with an LLOQ of $12.3 \mathrm{pg} / \mathrm{ml}$ [20].

Hence, while several studies report IL-17 levels in serum and cerebrospinal fluid (CSF), the majority of assays still lack the sensitivity and isoform selectivity further needed for a reliable detection of IL-17AA and IL-17FF in normal and disease state biofluids. Signal specificity and the differentiation from background signal in the relevant matrix is of prime importance when measuring low-abundance analytes, but such information is generally lacking in previously published reports.

Here, we have developed sensitive immunoassays using the Singulex Erenna ${ }^{\circledR}$ platform. These assays utilize well-characterized antibody reagents that specifically react with IL-17AA and IL-17FF, and were qualified using appropriate biological matrices. In this study, we used these assays to determine levels of IL17AA and IL-17FF in serum and CSF in normal, RA and relapsing remitting multiple sclerosis (RRMS) subjects to elucidate the relationship between IL-17AA and IL-17FF in systemic and CNS compartments. These results shed light on IL-17 regulatory mechanisms in both RA and RRMS disease populations and highlight the value of sensitive assays that distinguish between the various IL-17 isoforms.

\section{Material \& methods}

IL-17AA, IL-17FF \& IL-17AF production

\section{\& characterization}

Human IL-17AA, IL-17FF and IL-17AF (Genentech CR094, lot 001 and Genentech CRO75, lot 002, respectively) were produced in Chinese hamster ovary cells and purified proteins were verified by 
$\mathrm{N}$-terminal sequencing and peptide mass fingerprinting. Functional dimers were formed spontaneously and evaluated by LC. Production of IL-17AF was done as previously described [20].

\section{Antibody reagents}

Monoclonal antibody (MAb) clones (MAb1 and MAb2) against IL-17-FF were derived from hybridoma cell lines. Both antibodies were produced from mice immunized with IL-17FF immunogen, and transiently expressed in Chinese hamster ovary cells harboring IgG2a $\kappa$ framework. IL-17AA monoclonal antibody (MAb3) was derived from a mouse IgG2a $\kappa$ hybridoma cell line. IL-17AA and IL-17FF captured antibodies, MAb3 and MAb1, respectively, were immobilized on magnetic particles (MPs) by Singulex (CA, USA). MPs coated at $25-\mu \mathrm{g} \mathrm{Ab} / \mathrm{mg}$ MP were used for sample immunodepletion in the assessment of specificity, and the MPs coated at $12.5-\mu \mathrm{g} \mathrm{Ab} / \mathrm{mg}$ MP were used as capture reagents in the final assay format. IL-17AA and IL-17FF detection antibodies, polyclonal antibody (pAb, goat) AF317NA (R\&D Systems) and Genentech MAb2, respectively, were conjugated to a detection fluorophore at Singulex. IL-17AA and IL-17FF standard diluent, Singulex 02-0067-00, was used to dilute the standard curve analytes. IL-17AA assay buffer $(600 \mathrm{mM} \mathrm{NaCl}$ with $0.5 \%$ Triton X (TX)-100; Singulex) was used to dilute MPs. IL-17FF assay buffer ( $450 \mathrm{mM} \mathrm{NaCl}$ with $0.5 \%$ TX-100; Singulex) or IL-17AA high-salt sample diluent $(2 \times$ assay buffer: $1200 \mathrm{mM} \mathrm{NaCl}$ with $1.0 \%$ TX-100; Singulex) was used for sample dilution. Wash buffer (Singulex, 02-0514-00) was used at $1 \times$ for all wash steps. Elution buffer (Singulex) was used to dissociate the detection antibody from the MPs.

\section{Software, materials \& equipment}

Materials used in this evaluation included the following: 96-well microtiter plates for assay incubation (Pall, 5041); 96-well filter plates for sample and detection antibody filtration (E\&K Scientific, EK2470); 384-well plates for signal reading (Nunc, 264573); Jitterbug shaker (Boekel Scientific); and a HydroFlex plate washer (Tecan). Singulex Sgx link ${ }^{\mathrm{TM}}$ was used for statistical analysis and a Singulex SMDCurveFit ${ }^{\mathrm{TM}}$ algorithm was used for generation of standard curves and interpolation of data.

\section{Immunoassay procedure}

Ab-coated MPs were diluted to $50 \mu \mathrm{g} / \mathrm{ml}$ in assay buffer. Samples were prepared by diluting twofold into high-salt assay buffer. Diluted standard or sample $(100 \mu \mathrm{l})$ was mixed with $100 \mu \mathrm{l}$ of MPs ( $5 \mu \mathrm{g}$ MP per well) and incubated for $2 \mathrm{~h}$ at $25^{\circ} \mathrm{C}$, shaking in the Jitterbug (speed 5),
Table 1. Performance of IL-17AA and IL-17FF assays.

\begin{tabular}{lll}
\hline Parameter & IL-17AA & IL-17FF \\
LLOQ (pg/ml) & 0.1 & 0.1 \\
\hline Intra-assay \% CV: & & \\
- Low control & 13 & 7 \\
- Mid control & 15 & 9 \\
- High control & 3 & 1 \\
\hline Inter-assay \% CV: & & 16 \\
- Low control & 9 & 7 \\
- Mid control & 11 & 8 \\
- High control & 6 & \\
Spike recovery (\%): & & $98-107$ \\
- Serum & $88-120$ & $94-114$ \\
- CSF & 118 & $92-107$ \\
\hline Dilution linearity CSF (\%) & $82-120$ & \\
Cross-reactivity (\%): & & 0 \\
- IL-17AA & - & 1.5 \\
- IL-17FF & 0 & \\
- IL-17AF & 35 & \\
\hline CSF: Cerebrospinal fluid; CV: Coefficient of variation. & \\
\hline
\end{tabular}

followed by a single wash. The IL-17AA or IL-17FF detection antibodies were added at a final concentration of $500 \mathrm{ng} / \mathrm{ml}$ in assay buffer for IL-17AA and $100 \mathrm{ng} / \mathrm{ml}$ in assay buffer for IL-17FF. The MPs were transferred from the initial 96-well assay plate to a new plate to avoid eluting any nonspecific plate-bound detection reagent. The IL-17AA- and/or IL-17FF-specifically-bound detection reagent was then eluted with $10 \mu$ l elution buffer and detected by single molecule counting using the Erenna ${ }^{\circledR}$ system. All assays were performed at Singulex. To determine signal specificity in serum or CSF, Ab-coated MPs were prepared by binding capture antibody at $25-\mu \mathrm{g} / \mathrm{mg}$ MP. Samples were diluted 1:2 in high-salt assay buffer (125 $\mu \mathrm{l}$ sample in $125 \mu \mathrm{l} 2 \times$ assay buffer), then incubated with MP at $20 \mu \mathrm{g}$ MP/100 $\mu \mathrm{l}$ sample. The depletion plate was incubated for $1 \mathrm{~h}$ on a shaker at $25^{\circ} \mathrm{C}$. At the end of the incubation, the depletion plate was placed on a magnetic plate for $2 \mathrm{~min}$ to separate the MPs. Sample supernatant was transferred to the assay plate in duplicate $(100 \mu \mathrm{l} /$ well) for further analysis.

\section{Preparation of quality control samples for IL-17AA \& IL-17FF}

NHS pools were used as quality control (QC) samples. The low QC and mid-QC were from tested samples that had low or middle levels of endogenous cytokine, respectively. The high QC was prepared from pooled NHS that was spiked with recombinant analyte to approximately $20 \mathrm{pg} / \mathrm{ml}$ analyte. 


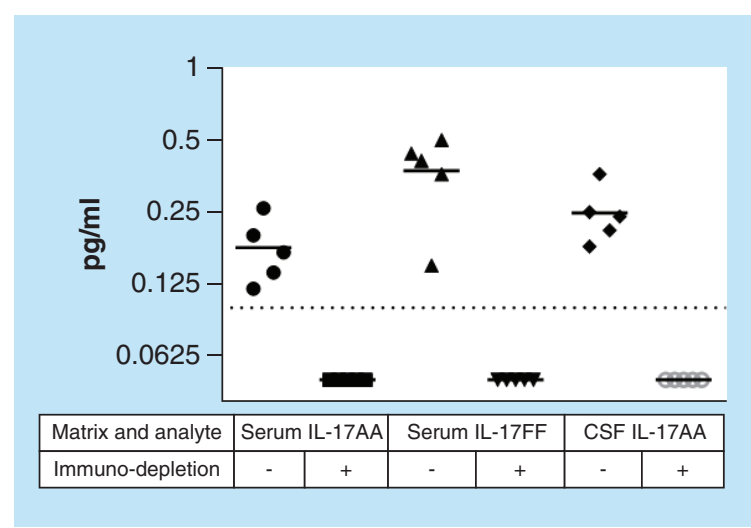

Figure 1. IL-17AA and IL-17FF assay specificity by immuno-depletion. IL-17AA and IL-17FF were measured in human serum (3 normal healthy subjects and 2 RA). IL-17AA was also tested in human CSF (5 normal healthy subjects). Dotted line represents LLOQ. Values below LLOQ were imputed to $0.05 \mathrm{pg} / \mathrm{ml}$. IL-17FF was not detected in CSF samples in this experiment. CSF: Cerebrospinal fluid.

\section{Determination of assay range}

A 12-point calibration curve was prepared for each run in Singulex standard assay diluent. The reference standard was diluted to a final working concentration of $25 \mathrm{pg} / \mathrm{ml}$ for both IL-17FF and IL-17AA assays. Data were obtained using Singulex Sgx Link software and analyzed using the SMDCurveFit algorithm. The LODs for the standard curves in standard diluent were determined by $2 \times$ (the standard deviation of background)/(slope of the curve) on the day the assay was run. The LLOQ was determined by adding exogenous recombinant standards to individual samples and evaluating acceptable recovery as that within $\pm 20 \%$ of the nominal concentration.

\section{Assessment of linearity \& recovery}

Recombinant IL-17AA or FF was spiked $(6.25 \mathrm{pg} / \mathrm{ml})$ into CSF samples to determine the recovery and linearity of dilution. The spiked samples were serially diluted in twofold steps in assay diluent to $1: 8$. The samples were then diluted twofold in high-salt assay buffer before testing. For serum samples, the aliquots were analytedepleted, and then spiked with IL-17AA or IL-17FF to final concentrations of $0.1,0.2,0.3,1$ and $10 \mathrm{pg} / \mathrm{ml}$. Spike recovery was calculated as follows: measured mean ([IL-17AA] - endogenous [IL-17AA])/expected spike $($ IL-17AA) $\times 100$. Linearity of dilution was calculated as follows: measured mean (IL-17AA) $\times 2 /$ concentration of the previous dilution $\times 100$.

\section{Assessment of assay precision}

Intra-assay precision was determined by measuring the QC samples. For intra-assay precision, six replicates from three separate controls were evaluated. Inter-assay precision was evaluated in three assay runs over 3 days, using three controls and two replicates.

\section{Evaluation of assay specificity}

Quantitation of IL-17FF in the AA assay, IL-17AA in the FF assay and IL-17A/F in both the AA and FF assays was evaluated by spiking various concentrations of the analyte of interest into assay diluent and calculating the percent cross-reactivity. In addition, specific signal was evaluated by the addition of recombinantspecific analyte to standard diluent or matrix samples and then immunodepleted with analyte-specific MPs or nonspecific MPs. Signal specificity was also evaluated by analyte-specific MP depletion of endogenous analyte in normal CSF, NHS and RA serum.
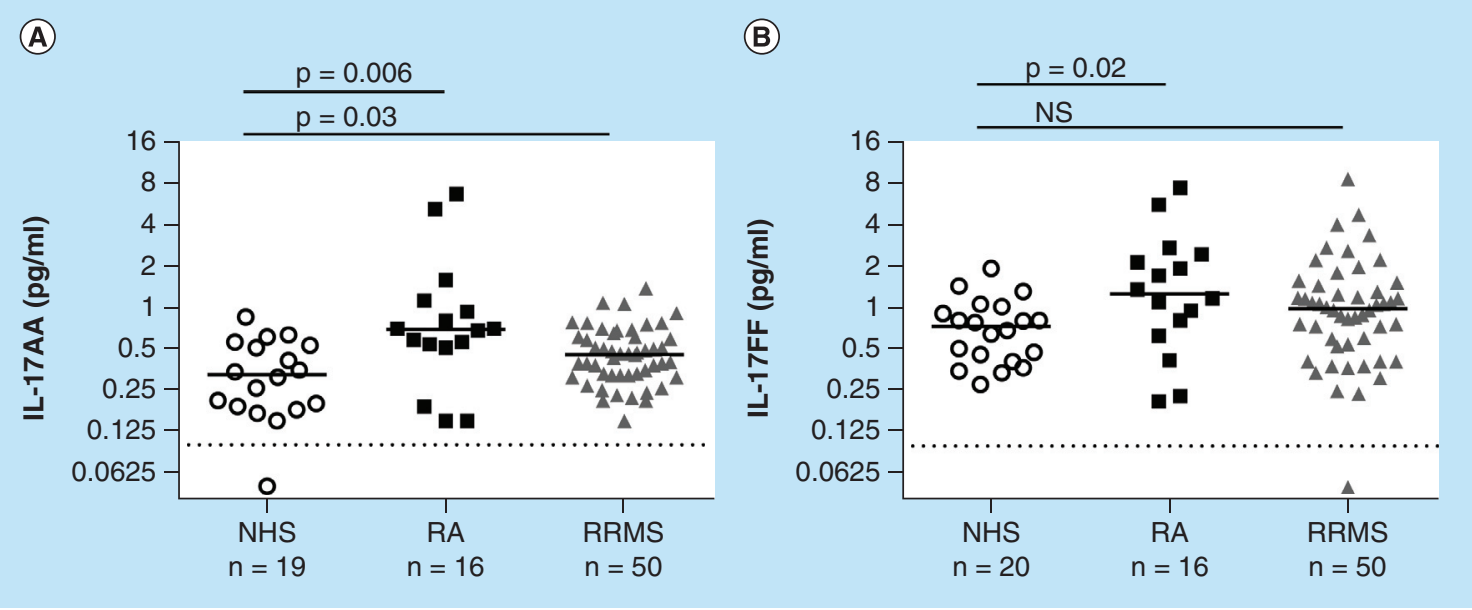

Figure 2. Serum levels of IL-17AA (A) and IL-17FF (B) in normal healthy subjects, rheumatoid arthritis and relapsing-remitting multiple sclerosis patients. Dashed line denotes dilution corrected LLOQ. Mann-Whitney test was performed to compare between levels in NHS and disease state. Values below LLOQ were imputed to $0.05 \mathrm{pg} / \mathrm{ml}$. NS: Not significant; NHS: Normal healthy subjects; RA: Rheumatoid arthritis; RRMS: Relapsing-remitting multiple sclerosis. 
Samples \& sample analysis

Serum samples from NHS were obtained from PrecisionMed, Inc. (CA, USA) and from BioreclamationIVT (NY, USA) (Supplementary Table 8A). Serum samples from RA patients were obtained from BioreclamationIVT (Supplementary Table 8B). Serum and CSF samples from 50 RRMS patients were obtained from PrecisionMed, Inc. (Table 4 \& Supplementary Table 9). Normal CSF was obtained from PrecisionMed, Inc. and BioreclamationIVT (Supplementary Table 8A [rows 11-20] \& 9). Serum samples were filtered using a $0.2-\mu \mathrm{m}$ filter plate. Samples were diluted 1:2 in high-salt assay buffer and in some cases subjected to analyte depletion. Nondepleted samples were treated similarly to depleted samples (i.e., diluted and placed on a shaker during the incubation for analyte depletion). Heterophilic antibody inhibitors were included in all assays.

\section{Results}

\section{Development \& characterization of IL-17AA \\ \& IL-17FF assays}

Final assay characteristics for IL-17AA and IL-FF are summarized in Table 1. Representative standard curves illustrate the linearity achieved at the sub-pg/ml level (Supplementary Figure 1 \& Supplementary Table 1) for both the IL-17AA and IL-17FF assays. Inter-assay precision for both assays was acceptable by the same criterion, with ranges of $3-15$ and $1-9 \%$, respectively (Supplementary Table 2). Intra-assay precision of IL-17AA and IL-17FF ranged from 6-11 and $7-16 \% \mathrm{CV}$, respectively. Accuracy was assessed in analyte-depleted samples of NHS CSF and in sera from patients. Sera from NHS and RA patients $(\mathrm{n}=5)$, as well as CSF from NHS patients $(\mathrm{n}=5)$, met the criteria of $\pm 20 \%$ recovery from nominal at all levels tested (Supplementary Table 3). Given the acceptable recovery data, the LLOQ in the matrix was set at $0.1 \mathrm{pg} / \mathrm{ml}$ for both IL-17AA and IL-17FF (Table 1). Normal CSF samples were evaluated for linearity and all samples and dilutions met the acceptability criterion by being within $\pm 20 \%$ of the previous dilution, and recoveries were within $\pm 20 \%$ of nominal (Supplementary Table 4). No cross-reactivity was detected between the IL-17AA and the IL-17FF assays (Supplementary Table 5). Minimal cross-reactivity $(1.5 \%)$ to IL-17AF was detected in the IL-17FF assay at concentrations of up to $10 \mathrm{pg} / \mathrm{ml}$. There was $35 \%$ cross-reactivity when IL-17AF was measured in the IL-17AA assay at $10 \mathrm{pg} / \mathrm{ml}$ (Supplementary Table 5). This was most likely due to the assay configuration utilizing a polyclonal antibody for detection.

To determine signal specificity, serum that was spiked in with IL-17AA or IL-17FF (Supplementary Table 6)
Table 2. IL-17AA serum levels in normal healthy subjects, rheumatoid arthritis and relapsing remitting multiple sclerosis patients.

\begin{tabular}{llll}
\hline Parameter & NHS & RA & RRMS \\
\hline $\mathrm{n}$ & 19 & 16 & 50 \\
\hline Minimum & 0.10 & 0.15 & 0.15 \\
\hline Q1 & 0.19 & 0.52 & 0.32 \\
\hline Median & 0.32 & 0.69 & 0.46 \\
Q3 & 0.54 & 1.07 & 0.62 \\
\hline Maximum & 0.85 & 6.68 & 1.38 \\
\hline Mean & 0.36 & 1.3 & 0.50 \\
\hline Lower $95 \% \mathrm{Cl}$ & 0.26 & 0.33 & 0.43 \\
Upper $95 \% \mathrm{Cl}$ & 0.47 & 2.30 & 0.57 \\
\hline
\end{tabular}

Values are in $\mathrm{pg} / \mathrm{ml}$. Samples were tested in duplicates.

NHS: Normal healthy subject; Q: Quartile; RA: Rheumatoid arthritis; RRMS: Relapsing remitting multiple sclerosis.

as well as serum and CSF samples containing endogenous IL-17 (Figure 1) was immunodepleted of specific analyte signal using MP-specific antibody particles and compared with nondepleted samples. Depleted matrix values quantitated below the LLOQ for both assays, confirming the specificity of the detected signal (Figure 1).

\section{IL-17AA \& IL-17FF levels in sera from NHS, RA \& RRMS patients}

IL-17AA and IL-17FF were determined in serum samples from NHS, RA and RRMS patients (Figure 2, Tables 2 \& 3; see Supplementary Tables 8 \& 9 for detailed demographic information). Out of the total 86 samples that were tested, only 2 were below LLOQ. Median serum levels of IL-17AA and IL-17FF in

Table 3. IL-17FF serum levels in normal healthy subjects, rheumatoid arthritis and relapsing remitting multiple sclerosis patients

\begin{tabular}{|llll|}
\hline Parameter & NHS & RA & RRMS \\
\hline Minimum & 19 & 16 & 50 \\
\hline Q1 25\% & 0.28 & 0.21 & 0.10 \\
\hline Median & 0.42 & 0.68 & 0.55 \\
\hline Q3 & 0.74 & 1.27 & 0.99 \\
\hline Maximum & 1.00 & 2.39 & 1.48 \\
\hline Mean & 1.95 & 7.54 & 8.75 \\
\hline Lower $95 \% \mathrm{Cl}$ & 0.77 & 1.95 & 1.34 \\
Upper $95 \% \mathrm{Cl}$ & 0.57 & 0.88 & 0.94 \\
\hline Vav a & 0.98 & 3.02 & 1.76 \\
\hline
\end{tabular}

Values are in $\mathrm{pg} / \mathrm{ml}$. Samples were tested in duplicates.

NHS: Normal healthy subject; Q: Quartile; RA: Rheumatoid arthritis; RRMS: Relapsing remitting multiple sclerosis. 


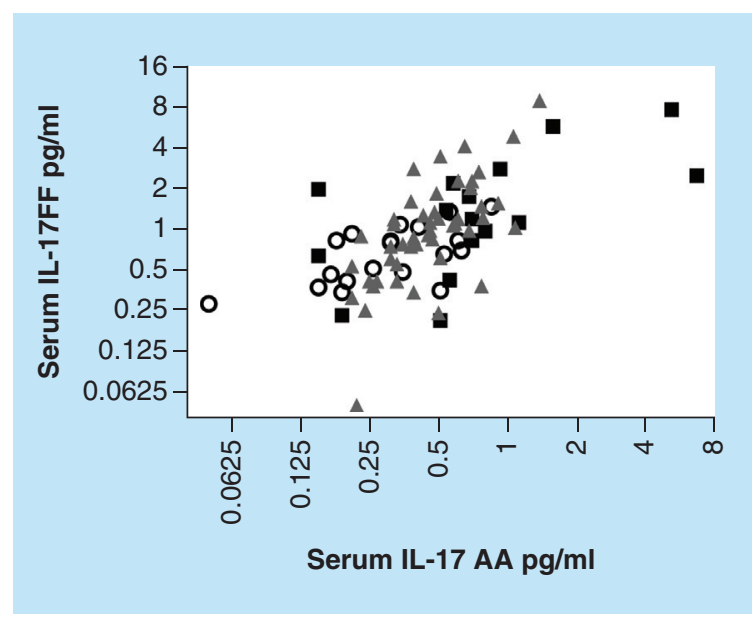

Figure 3. Correlation between serum levels of IL-17AA and IL-17-FF in normal healthy subjects, rheumatoid arthritis and relapsing-remitting multiple sclerosis patients.

o NHS: Normal healthy subjects; - RA: Rheumatoid arthritis; $\triangle$ RRMS: Relapsing-remitting multiple sclerosis.

Spearman $r=0.63 ; p$-value $=<0.0001$

NHS were 0.32 and $0.74 \mathrm{pg} / \mathrm{ml}$, respectively. Minimum and maximum values in NHS were $0.1-0.85$ and $0.28-1.95 \mathrm{pg} / \mathrm{ml}$ for IL-17AA and IL-17FF, respectively.

Serum levels of IL-17AA and IL-17FF were significantly higher in samples from RA patients as compared with NHS with median values of 0.69 and $1.27 \mathrm{pg} / \mathrm{ml}$, respectively. Serum levels of IL-17AA were also significantly elevated in RRMS patients as compared with NHS (median of $0.46 \mathrm{pg} / \mathrm{ml}$ in RRMS as compared with $0.32 \mathrm{pg} / \mathrm{ml}$ in NHS) but were not significantly different in IL-17FF levels; albeit, a strong trend for increased levels in RRMS as compared with NHS (median of $0.99 \mathrm{pg} / \mathrm{ml}$ in RRMS as compared with $0.74 \mathrm{pg} / \mathrm{ml}$ in NHS) (Figure 2, Tables $2 \& 3$ ).

Despite the difference in absolute levels of IL-17AA and IL-17FF in serum, a strong correlation was observed between the two isoforms (Figure 3). This correlation was consistent across all samples from both NHS and disease state and throughout the value range (Spearman $\mathrm{r}=0.63$; $\mathrm{p}$-value $\leq 0.0001)$.

\section{IL-17AA \& IL-17FF levels in CSF from RRMS \& matched NHS}

IL-17AA and IL-17FF levels were determined in CSF samples from RRMS subjects $(\mathrm{n}=50)$ and matched NHS $(\mathrm{n}=10)$ (Figure 4 , see Table 4 \& Supplementary Table 9 for additional demographic information). In general, levels of both IL-17 isoforms were lower in CSF compared with serum levels and of the 60 CSF samples tested (10 NHS and 50 RRMS) 13/60 registered below LLOQ for IL-17AA and 43/60 registered below LLOQ for IL-17FF. In NHS, only 1/10 CSF samples had IL-17FF levels that registered within the quantitative range. In contrast to serum, both NHS and RRMS CSF samples contained higher levels of IL-17AA compared with IL-17FF. In RRMS, the mean concentration of IL-17AA was $0.42 \mathrm{pg} / \mathrm{ml}$ and the mean concentration of IL-17FF was $0.07 \mathrm{pg} / \mathrm{ml}$ in CSF whereas in serum the mean concentration of IL-17AA was $0.5 \mathrm{pg} / \mathrm{ml}$ and the mean concentration of IL-17FF was $1.34 \mathrm{pg} / \mathrm{ml}$ (Tables 2-3 \& Supplementary Table 7).

Although CSF levels of IL-17AA were significantly higher in samples from RRMS patients as compared with NHS (median values of 0.38 and $0.17 \mathrm{pg} / \mathrm{ml}$, respectively), CSF levels of IL-17FF were very low
(A)

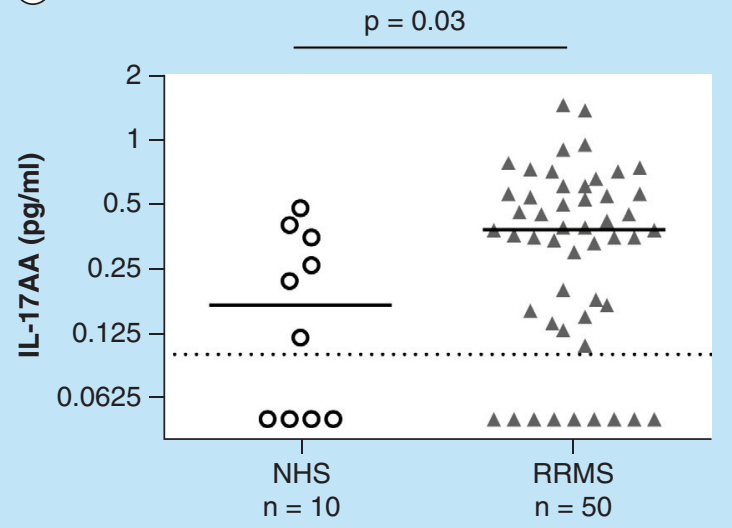

(B)

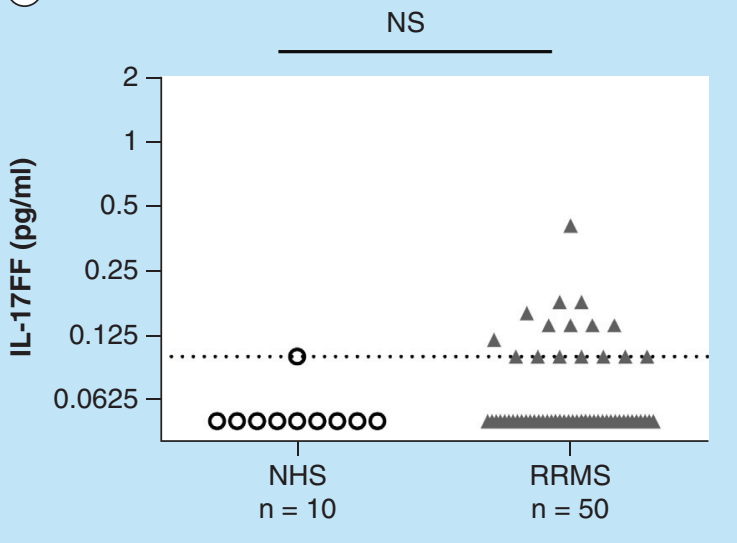

Figure 4. Cerebrospinal fluid levels of IL-17AA (A) and IL-17FF (B) in matched normal healthy subjects and relapsing-remitting multiple sclerosis patients. Dashed line denotes dilution corrected LLOQ. Mann-Whitney test was performed to compare between levels in NHS and disease state. Values below LLOQ were imputed to $0.05 \mathrm{pg} / \mathrm{ml}$. NHS: Normal healthy subjects; NS: Not significant; RRMS: Relapsing-remitting multiple sclerosis. 
Table 4. Clinical and demographic profile of relapsing remitting multiple sclerosis and normal healthy subjects.

\begin{tabular}{|c|c|c|}
\hline Demographic parameter & RRMS $(n=50)$ & Control $(n=10)$ \\
\hline \multicolumn{3}{|l|}{ Age (year): } \\
\hline - Mean (SD) & $50.7(11)$ & $52.3(6.3)$ \\
\hline - Median & 52.5 & 49.5 \\
\hline - Range & $28-73$ & $44-62$ \\
\hline \multicolumn{3}{|l|}{ Gender ( $\mathrm{n}[\%$ of total]): } \\
\hline - Female & $38(76)$ & $5(50)$ \\
\hline \multicolumn{3}{|c|}{ Race and ethnicity (n [\% of total]): } \\
\hline - Asian & $1(2)$ & $0(0)$ \\
\hline - Black & $2(4)$ & $1(10)$ \\
\hline - Caucasian & $45(90)$ & $9(90)$ \\
\hline - Hispanic & $2(4)$ & $0(0)$ \\
\hline \multicolumn{3}{|l|}{ Smoker (n [\% of total]): } \\
\hline- No & $16(32)$ & $7(70)$ \\
\hline \multicolumn{3}{|c|}{ Anti-inflammatory medications ( $[\%$ of total]): } \\
\hline- No & $45(90)$ & $10(100)$ \\
\hline \multicolumn{3}{|c|}{ MS medications ( $\mathrm{n}[\%$ of total]): } \\
\hline- No & $15(30)$ & $10(100)$ \\
\hline \multicolumn{3}{|l|}{ MMSE score: } \\
\hline - Mean (SD) & $27.5(2)$ & $29.2(0.8)$ \\
\hline - Median & 28 & 29 \\
\hline - Range & $21-30$ & $28-30$ \\
\hline
\end{tabular}

across the sample set and therefore there was no significant difference between RRMS and NHS (Figure 4B). However, more samples registered within the quantitative range for IL-17FF from RRMS patients (32\%) as compared with NHS (10\%) suggesting a trend toward increased levels of IL-17FF in CSF from RRMS patients.

Interestingly, although there is substantial fluid exchange between the blood and the CSF compartments, no correlation was found between IL-17AA serum levels and CSF levels in either NHS or RRMS patients (Figure 5).

\section{Conclusion}

The growing interest in IL-17 as a therapeutic target for autoimmune disorders prompted the development of immunoassays to better understand the relationship between IL-17 levels and clinical manifestation. T-cell derived IL-17 cytokines, IL-17AA and IL-17FF, are released and act at sites of inflammation such as the synovial fluid in RA and the CNS in MS. These compartments are often inaccessible to sampling, and
IL-17 levels are primarily determined in circulation. Hence, increasing our understanding of the relationship between IL-17 levels at sites of inflammation and circulating levels can further highlight the role of IL-17 isoforms in autoimmunity.

Low circulating levels of IL-17 combined with the presence of multiple isoforms pose a bioanalytical challenge. Although several studies report IL-17 levels in serum and CSF, the majority of assays still lack the sensitivity and isoform selectivity further needed for a reliable detection of IL-17AA and IL-17FF in normal and disease state biofluids. Signal specificity and the differentiation from background signal in the relevant matrix is of prime importance when measuring lowabundance analytes but such information is generally lacking in previously published reports. IL-17 isoforms cannot be robustly quantified with a typical ELISA platform as was demonstrated by Deforge $e t$ al. due to sensitivity limitations [20]. Recently, reference values for serum IL-17AA in healthy subjects were reported using a high-sensitivity platform demonstrating that the majority of values registered at the sub-pg/ml level [24]. 


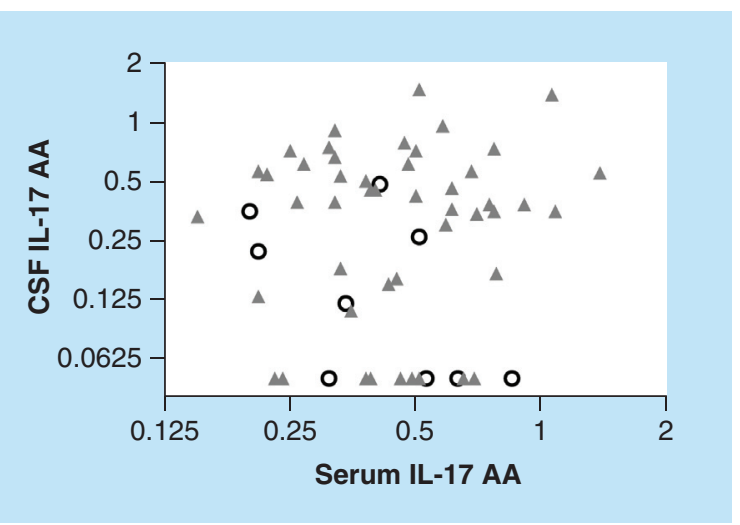

Figure 5. Relationship of serum and cerebrospinal fluid levels of IL-17AA in normal healthy subject and relapsing-remitting multiple sclerosis patients.

- NHS: Normal healthy subjects;

$\triangle$ RRMS: Relapsing-remitting multiple sclerosis. Spearman $r$ not significant.

This observation puts previous reports into question using partially characterized immunoassays with insufficient sensitivity and specificity to detect circulating IL-17. For example, in our view, it is impossible to accurately determine serum IL-17 levels in healthy controls that are present at sub-pg/ml levels, using an ELISA assay with an LLOQ of $4.6 \mathrm{pg} / \mathrm{ml}$ as reported by Arican et al. [22]. Hence, we propose that a combination of a high-sensitivity immunoassay platform coupled with robust assay qualification is required for the reliable measurement of IL-17 in the circulation and CSF. Here, we described the development and qualification of IL-17AA and IL-17FF assays using well-characterized antibody reagents and well-qualified standards combined with the ultrasensitive Erenna Immunoassay System. Taking into account the expected low levels of IL-17AA and IL-17FF, reagent specificity and matrix effects were rigorously characterized in order to increase accuracy and reliability of the quantitative readout. We implemented an immune-depletion strategy in order to determine precisely how much signal resulted from endogenous IL-17AA and IL-17FF in both CSF and serum. Additionally, to reduce background signal and obtain accurate readout at the sub-pg/ml level, samples were diluted in high-salt buffer prior to incubation with Ab-conjugated MP beads. Importantly, we found no cross-reactivity between IL-17AA and IL-17FF in either assay. The IL-17FF assay also showed no crossreactivity with IL-17AF; however, in the IL-17AA assay, we did detect up to $35 \%$ cross-reactivity with IL-17AF. Although some cross-reactivity was detected between IL-17AA and 1L-17FF, due to the strong correlation between IL-17AA and IL-17FF observed in serum (Figure 3) it is unlikely that IL-17AF levels had a marked impact on the values observed with the IL-17AA assay.
Using these well-qualified immunoassays, we were able to detect IL-17AA and IL-17FF in sera from RA and RRMS patients and the healthy controls. In addition, to our knowledge, this is the first time that IL-17AA and IL-17FF have been reliably measured in CSF from RRMS patients and compared with matched healthy controls.

In NHS $(\mathrm{n}=19)$, the median value of circulating serum IL-17AA was $0.32 \mathrm{pg} / \mathrm{ml}$ (range $0.1-0.85 \mathrm{pg} / \mathrm{ml}$ ), whereas serum IL-17FF was $0.74 \mathrm{pg} / \mathrm{ml}$ (range $0.28-1.95 \mathrm{pg} / \mathrm{ml}, \mathrm{n}=85$ ). Previous studies reported a wide range of IL-17A levels in normal serum, varying from undetectable to approximately $30 \mathrm{pg} / \mathrm{ml}$. Those values were obtained using commercially available ELISAs with reported LLOQs of 4.6 and $4 \mathrm{pg} / \mathrm{ml}$, respectively [22,23]. Although it is possible that the use of different IL-17 standards can account for such differences it also highlights some of the challenges and inconsistencies in accurately determining IL-17 levels

In serum samples from RA patients $(n=16)$, both IL-17AA and IL-17FF levels were significantly elevated as compared with NHS with medians of 0.69 and $1.27 \mathrm{pg} / \mathrm{ml}$ for IL-17AA and IL-17FF, respectively. These IL-17AA values are consistent with a recently published report by Jain et al. using a Singulex assay kit [25]. In contrast, the IL-17FF values reported by Jain $e t$ al. are markedly higher than our current finding (in NHS: $4.4 \mathrm{pg} / \mathrm{ml}$ as compared with $0.74 \mathrm{pg} / \mathrm{ml}$ in the current study; and in RA: $81 \mathrm{pg} / \mathrm{ml}$ compared with $1.27 \mathrm{pg} / \mathrm{ml}$ in the current study). Since the IL-17FF assay described in the Jain et al. report did not provide information regarding signal specificity, the possibility exist that some of the IL-17FF signal was nonspecific. Indeed, Singulex has recently introduced a second generation of the IL-17FF assay kit that is consistent with our current data and reports a median value of $0.83 \mathrm{pg} / \mathrm{ml}$ for IL-17FF in plasma from 20 healthy donors [26].

Analysis of serum samples from RRMS patients revealed elevated levels of IL-17AA as compared with NHS (median $0.46 \mathrm{pg} / \mathrm{ml}(\mathrm{n}=50)$ and $0.32 \mathrm{pg} / \mathrm{ml}$ $(n=19)$, respectively). Serum IL-17FF levels in RRMS patients were not significantly different from NHS; however, there was a trend toward increased levels (a median of $0.99 \mathrm{pg} / \mathrm{ml}$ vs $0.74 \mathrm{pg} / \mathrm{ml}$ in RRMS and NHS, respectively). Although absolute levels of serum IL-17FF were generally higher than IL-17AA levels, there was a strong correlation between serum IL-17AA and serum IL-17FF across the entire sample set (NHS, RA and RRMS samples) suggesting similar production and/or turn-over kinetics for these isoforms in serum in both healthy state and during inflammatory disease. Interestingly, and in contrast to serum, IL-17AA was found to be the predominant isoform in CSF as compared with markedly lower levels of IL-17FF in this compartment (a median of 0.38 
vs $0.05 \mathrm{pg} / \mathrm{ml}$, respectively). This may indicate that the synthesis and/or the clearance mechanisms of these isoforms in the CNS may be distinct from those governing circulating levels. Consistent with this notion, no correlation was detected between serum and CSF levels of IL-17AA in NHS and RRMS patients. One possibility is that different cell populations are responsible for IL-17 production in circulation and in the central compartment. This hypothesis has been further supported by a recent report suggesting that although mRNA expression levels of IL-17AA and IL-17FF strongly correlate in gut ulcers, their pattern of expression during disease progression was markedly different and may reflect a differential role of these isoforms in Th17 responses [27]. Importantly, heterogeneous expression of the different IL-17 ligands and their receptors may provide a possible explanation for inadequate or nonresponse to anti-IL-17 therapy [8]. Understanding the cues associated with circulating IL-17 levels may be used to better characterize the patient population and thereby optimize anti-IL-17 therapy in autoimmunity.

\section{Future perspective}

Robust measurement of low-abundance biomarkers has the potential to provide valuable diagnostic and prognostic information. To do that reliably, it is essential to conduct a careful characterization of the sensitivity and specificity of a given assay. The development of IL-17AA and IL-17FF assays described here provides insight into some of the challenges associated with detection of low-level analytes in complex matrices. Measuring the levels of IL-17AA and IL-17FF isoforms in different compartments (e.g., serum, and CSF) may shed further light on the regulation of IL-17 isoforms in various microenvironments and will enable better biological and clinical interpretation of circulating IL-17 levels. As the cohort size used in this study does not permit a definitive substantiation of such relationship, additional work is needed to enable the use of circulating IL-17 as a diagnostic and/or prognostic biomarker in inflammatory diseases.

Further use of robust, high-sensitivity quantitation techniques will provide additional insight into the role

\section{Executive summary}

\section{Background}

- Th17 derived IL-17 isoforms are secreted at sites of inflammation and are known to play a role in autoimmunity.

- The ability to reliably measure IL-17 isoforms in accessible circulating body fluids such as serum and cerebrospinal fluid (CSF) may hold important information about their specific role in autoimmune diseases. However, circulating IL-17 is present at the sub-pg/ml levels thereby posing a bioanalytical challenge.

- Although it is known that Th17 are activated during autoimmunity, the relative levels and relationship of their effector cytokines IL-17AA and IL-17FF in systemic and central compartments are not well understood and can shed further light on the involvement of these isoforms in different autoimmune diseases such as rheumatoid arthritis (RA) and relapsing remitting multiple sclerosis (RRMS).

\section{Experimental}

- IL-17AA and IL-17FF assays were developed using well-characterized antibody reagents coupled with the highly sensitive Singulex immunoassay system.

- Assays' specificity was confirmed through immune-depletion and assessment of cross-reactivity against each isoform as well as the heterodimer IL-17AF.

- IL-17 isoforms were determined in sera and CSF samples from a cohort of 50 RRMS patients as well as sera from RA patients, and compared with normal healthy subjects (NHS).

\section{Results \& discussion}

- IL-17AA and IL-17FF assays exhibited high specificity and sensitivity with an LLOQ of $0.1 \mathrm{pg} / \mathrm{ml}$.

- Levels of IL-17AA were significantly higher in serum from RA and RRMS patients as compared with NHS.

- Levels of IL-17FF were significantly higher in RA sera and showed a trend of increase in RRMS, as compared with NHS.

- There was a strong correlation between the two isoforms in serum from NHS, RA and RRMS patients.

- IL-17AA was also elevated in CSF from RRMS patients as compared with NHS; however, no correlation was observed between serum and CSF levels.

- Although serum IL-17FF levels were generally higher than IL-17AA, IL-17AA was found to be the predominant form in CSF.

\section{Conclusion}

- IL-17AA and IL-17FF are present at low levels in serum and CSF with the majority of values registering below $1 \mathrm{pg} / \mathrm{ml}$.

- A differential relationship between the levels of IL-17AA and IL-17FF was detected in circulation as compared with the central compartment.

- Such robust quantitation techniques provide an opportunity to further study the unique role of IL-17 isoforms in the pathogenesis and progression of inflammatory diseases. 
of other circulating low-abundance analytes in the progression of various disorders as was demonstrated recently [28] and will enable their use as informative biomarkers.

\section{Supplementary data}

To view the supplementary data that accompany this paper please visit the journal website at: www.future-science.com/ doi/full/10.4155/bio-2016-0207

\section{Acknowledgements}

The authors would like to thank Singulex colleagues for technical support. The authors would also like to thank J Good, $\checkmark$ Anania, A Morimoto and W Mathews at Genentech for technical support and review of the manuscript as well as the D-TECT Team at Genentech for supporting this study.

Financial \& competing interests disclosure

C Schofield, SK Fischer, MJ Townsend, S Mosesova, K Peng, AF Setiadi, A Song and A Baruch are employees of Roche. The

\section{References}

Papers of special note have been highlighted as:

of interest; $\bullet$ of considerable interest

1 Jin W, Dong C. IL-17 cytokines in immunity and inflammation. Emerg. Microbes Infect. 2(9), e60 (2013).

2 Korn T, Bettelli E, Oukka M, Kuchroo VK. IL-17 and Th17 cells. Annu. Rev. Immunol. 27, 485-517 (2009).

3 Hymowitz SG, Filvarof EH, Yi JP et al. IL-17s adopt a cystine knot fold: structure and activity of a noval cytokine, IL-17F, and implications for receptor binding. EMBO J. 20(19), 5332-5332 (2001).

4 Fujie $\mathrm{H}$, Niu $\mathrm{K}$, Ohba $\mathrm{M}$ et al. A distinct regulatory role of Th17 cytokines IL-17A and IL-17F in chemokine secretion from lung microvascular endothelial cells. Inflammation 35(3), 1119-1131 (2012).

5 Chang SH, Dong C. A novel heterodimeric cytokine consisting of IL-17A and IL-17F regulates inflammatory responses. Cell Res. 17(5), 435-440 (2007).

6 Pappu R, Ramirez-Carrozzi V, Sambandam A. The interleukin-17 cytokine family: critical players in host defence and inflammatory diseases. Immunology 134(1), $8-16$ (2011).

7 Milner JD. IL-17 producing cells in host defense and atopy. Curr. Opin. Immunol. 23(6), 784-788 (2011).

8 van Baarsen LG, Lebre MC, van der Coelen D et al. Heterogeneous expression pattern of interleukin 17A (IL$17 \mathrm{~A})$, IL-17F and their receptors in synovium of rheumatoid arthritis, psoriatic arthritis and osteoarthritis: possible explanation for nonresponse to anti-IL-17 therapy? Arthritis Res. Ther. 16(4), 426 (2014).

- Differential expression of the different IL-17 ligands and their receptors may provide a possible explanation for inadequate or nonresponse to anti-IL-17 therapy. authors have no other relevant affiliations or financial involvement with any organization or entity with a financial interest in of financial conflict with the subject matter or materials discussed in the manuscript apart from those disclosed.

Writing assistance was utilized in the production of this manuscript. Genentech, Inc. provided support for third-party editorial assistance by Anshin Biosolutions for this manuscript.

\section{Ethical conduct of research}

The authors state that they obtained appropriate institutional review board approval or have followed the principles outlined in the Declaration of Helsinki for all human or animal experimental investigations. In addition, for investigations involving human subjects, informed consent has been obtained from the participants involved.

\section{Open access}

This work is licensed under the Creative Commons Attribution 4.0 License. To view a copy of this license, visit http:// creativecommons.org/licenses/by/4.0/

9 Gaffen SL. Structure and signalling in the IL-17 receptor family. Nat. Rev. Immunol. 9(8), 556-567 (2009).

10 Miossec P, Korn T, Kuchroo VK. Interleukin-17 and type 17 helper T cells. N. Engl. J. Med. 361(9), 888-898 (2009)

11 Beringer A, Noack M, Miossec P. IL-17 in chronic inflammation: from discovery to targeting. Trends $\mathrm{Mol}$. Med. 22(3), 230-241 (2016).

12 Tzartos JS, Friese MA, Craner MJ et al. Interleukin-17 production in central nervous system-infiltrating $\mathrm{T}$ cells and glial cells is associated with active disease in multiple sclerosis. Am. J. Pathol. 172(1), 146-155 (2008).

13 Kellner H. Targeting interleukin-17 in patients with active rheumatoid arthritis. Ther. Adv. Musculoskel. Dis. 5(3), 141-152 (2013).

- Clinical as well as experimental evidence suggests that IL-17A (also known as IL-17) is an attractive therapeutic target in rheumatoid arthritis.

14 Spuls PI, Hooft L. Brodalumab and ixekizumab, antiinterleukin-17-receptor antibodies for psoriasis: a critical appraisal. Br. J. Dermatol. 167(4), 710-713 (2012).

- IL-17RA antibody therapy showed significant reduction in the Psoriasis Area Severity Index when both therapies were compared with placebo.

15 Langley RG, Elewski BE, Lebwohl M et al. Secukinumab in plaque psoriasis - results of two Phase 3 trials. N. Engl. J. Med. 371(4), 326-338 (2014).

- $\quad$ Phase III studies validate IL-17A as an important therapeutic target in moderate-to-severe plaque psoriasis.

16 Elain G, Jeanneau KR, Rutkowska A, Mir AK, Dev KK. The selective anti-IL17A monoclonal antibody secukinumab (AIN457) attenuates IL17A-induced levels of IL6 in human astrocytes. Glia 62(5), 725-735 (2014). 

level and interferon beta response in patients with multiple sclerosis. JAMA Neurol. 70 (8), 1017-1021 (2013).

- Highlights the specific unmet need for biomarkers that predict therapeutic response.

18 Bălașa R, Bajko Z, HuṬanu A. Serum levels of IL-17A in patients with relapsing-remitting multiple sclerosis treated with interferon-beta. Mult. Scler. 19(7), 885-890 (2013).

- Relapsing remitting multiple sclerosis patients with high IL-17A levels do not respond well to IFN- $\beta$ therapy, LOD $1.6 \mathrm{pg} / \mathrm{ml}$.

19 Ramos-Cejudo J, Oreja-Guevara C, Aroeira LS, Rodrigues de Antonio L, Chamorro B, Diez-Tejedor E. Treatment with natalizumab in relapsing-remitting multiple sclerosis patients induces changes in inflammatory mechanism. J. Clin. Immunol. 31(4), 623-631 (2011)

20 DeForge LE, Loyet KM, Delarosa D et al. Evaluation of heterophilic antibody blocking agents in reducing false positive interference in immunoassays for IL-17AA, IL-17FF and IL-17AF. J. Immunol. Method 362(1-2), 70-81 (2010).

- $\quad$ Robust characterization of IL-17AA and IL-17FF immunoassays. IL-17AA and IL-17FF are present in very low levels in circulation. This demonstrates the importance of evaluating false-positive signals and the need for more sensitive IL-17 assays.

21 Chen DY, Chen YM, Chen HH, Hsieh CW, Lin CC, Lan JL. Increasing levels of circulating Th17 cells and interleukin-17 in rheumatoid arthritis patients with an inadequate response to anti-TNF- $\alpha$ therapy. Arthritis Res. Ther. 13, R126 (2011).

22 Arican O, Aral M, Sasmaz S, Caragil P. Serum levels of TNF- $\alpha$, IFN- $\gamma$, IL-6, IL-8, IL-12, IL-17, and IL-18 in patients with active psoriasis and correlation with disease severity. Mediat. Inflamm. 2005(5), 273-279 (2005).

23 Lei Z, Liu G, Huang Q, Lv M, Zu R, Zhang GM et al. SCF and IL-31 rather than IL-17 and BAFF are potential indicators in patients with allergic asthma. Allergy 63(3), 327-332 (2008).

24 Todd J, Simpson P, Estis J, Torres V, Wub AH. Reference range and short- and long-term biological variation of interleukin (IL)-6, IL-17A and tissue necrosis factor-alpha using high sensitivity assays. Cytokine 64(3), 660-665 (2013)

- One of the examples available only in literature of robust characterization of IL-17AA, developing assay with the proper sensitivity level.

25 Jain M, Attur M, Furer V et al. Increased plasma IL$17 \mathrm{~F}$ levels in rheumatoid arthritis patients are responsive to methotrexate, anti-TNF, and T cell costimulatory modulation. Inflammation 38 (1), 180-186 (2015).

26 Singulex. www.singulex.com/

27 Iboshi Y, Nakamura K, Fukaura K et al. Increased IL-17A/IL-17F expression ratio represents the key mucosal $\mathrm{T}$ helper/regulatory cell-related gene signature paralleling disease activity in ulcerative colitis. J. Gastroenterol. doi:10.1007/s00535-016-1221-1 (2016) (Epub ahead of print).

- IL-17 AA and IL-17FF differ in their pattern of expression during progression of gut inflammation and may reflect differential role of these isoforms in Th17 responses.

28 Cai F, Hornauer H, Peng K, Schofield CA, Scheerens H, Morimoto AM. Bioanalytical challenges and improved detection of circulating levels of IL-13. Bioanalysis 8(4), 323-332 (2016). 